\title{
Down-regulation of glomerular matrix metalloproteinase-2 gene in human NIDDM
}

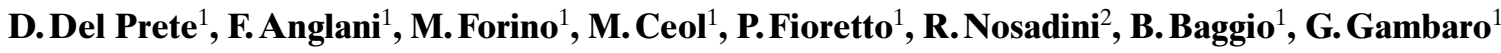 \\ ${ }^{1}$ Institute of Internal Medicine, University of Padova, Italy \\ ${ }^{2}$ Chair of Endocrinology, University of Sassari, Italy
}

\begin{abstract}
Summary Regulation of mesangial matrix deposition is a dynamic phenomenon involving synthetic and degradative processes. The latter involve a number of matrix metalloproteinases (MMP) and tissue inhibitors of matrix metalloproteinases (TIMP). Experimental studies suggest that mesangial matrix degradation is inhibited in diabetic nephropathy, and that this phenomenon has a pathogenic role. The expression of genes for MMP2 and TIMP2 in human diabetic nephropathy was investigated. Reverse transcription polymerase chain reaction was carried out in microdissected glomeruli and tubulo-interstitium obtained from kidney biopsies. We studied 16 NIDDM patients, 5 patients with glomerulonephritis or chronic kidney transplant rejection, and 5 normal control subjects. Albumin excretion rate and renal histology for NIDDM patients were available. Contrary to TIMP2 which was expressed both in tubulo-interstitium and glomeruli in almost all renal biopsies,
\end{abstract}

MMP2 gene down-regulation was observed in glomeruli from all NIDDM patients, irrespective of the albumin excretion rate, and of renal histology. In contrast, this gene was expressed in biopsies from other subjects $\left(\chi^{2}=20.6 ; p=0.000\right)$. In conclusion, this study demonstrates that: 1 ) in glomeruli of NIDDM patients the MMP2 gene is down-regulated; 2) in biopsies of NIDDM patients the MMP2/TIMP2 pattern is peculiar for NIDDM; 3) the MMP2 gene down-regulation is observed in all NIDDM patients, irrespective of the level of albuminuria and of renal histology. MMP2 gene down-regulation seems to be a molecular epiphenomenon of diabetes, rather than a marker of diabetic nephropathy. [Diabetologia (1997) 40: 1449-1454]

Keywords Metalloproteinases, MMP2, TIMP2, noninsulin-dependent diabetes mellitus, diabetic nephropathy, microalbuminuria
Mesangial matrix expansion is the hallmark of diabetic nephropathy. It is generally assumed that mesangial expansion in diabetes mellitus is due to the extracellular matrix enlargement rather than to mesangial cell hyperplasia. Metabolic studies in

Received: 9 April 1997 and in revised form: 21 July 1997

Corresponding author: G. Gambaro, M. D., Ph. D., Divisione di Nefrologia, Istituto di Medicina Interna, Policlinico Universitario, via Giustiniani 2, I-35128 Padova, Italy

Abbreviations: AER, Albumin excretion rate; MMP, matrix metalloproteinase; NIDDM, non-insulin-dependent diabetes mellitus; RT, reverse transcription; PCR, polymerase chain reaction; TGF- $\beta$, transforming growth factor- $\beta$; TIMP, tissue inhibitor of metalloproteinase experimental models have provided evidence that the enhanced production of collagen is a key event in the development of diabetic glomerular extracellular matrix abnormalities. However, regulation of mesangial matrix is a dynamic process involving synthetic as well as degradative processes. The latter involve a number of matrix metalloproteinases (MMP) and tissue inhibitors of matrix metalloproteinases (TIMP). Recently the issue of MMP activity in diabetic nephropathy has been addressed, and the idea has been proposed of reduced degradation as a mechanism concurring with the enlargement of the mesangial area; there are experimental data to support this hypothesis [1-4] and find in MMP2 a possible culprit of the anomalous degradation [1,4]. That MMP2 might be involved is conceivable since it specifically 
Table 1. Clinical data on NIDDM patients

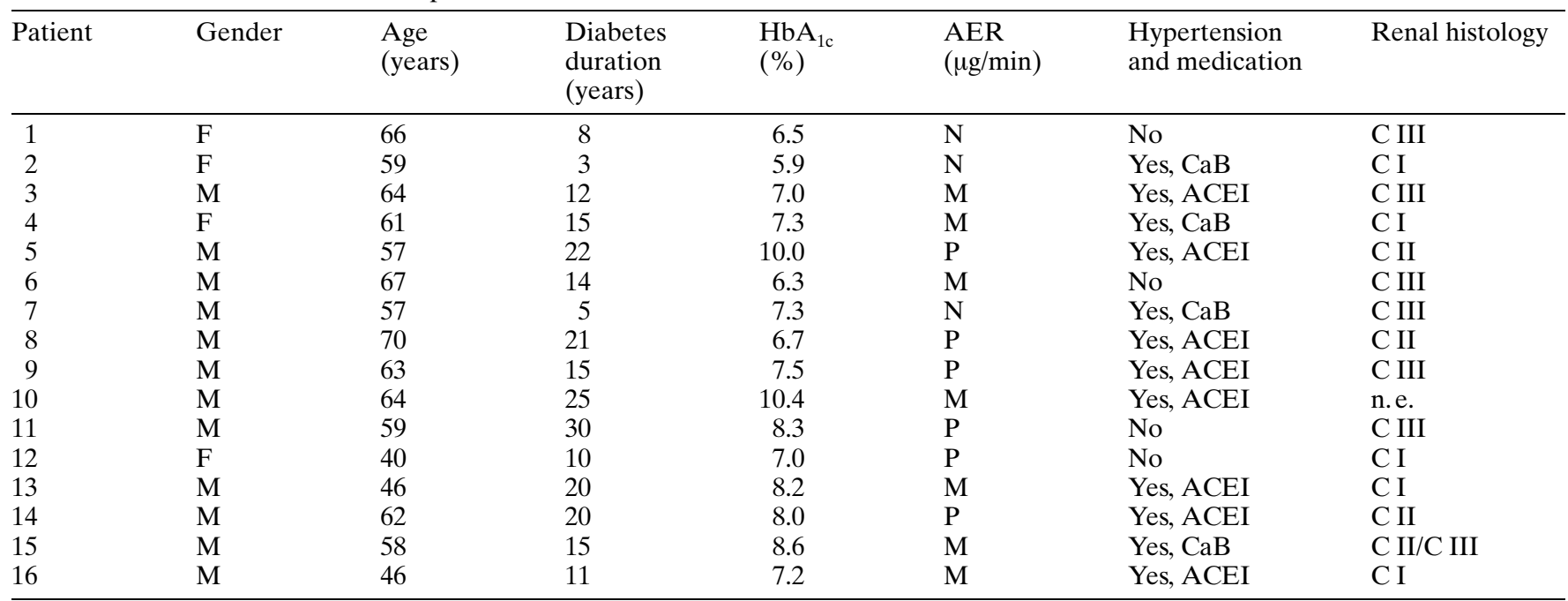

Patients reported in order of enrollment

n. e., Not evaluated due to lack of glomeruli in core tissue for microscopy

AER, N, Normal (<20 $\mu \mathrm{g} / \mathrm{min})$; M, microalbuminuria (20$200 \mu \mathrm{g} / \mathrm{min}$ ); P, macroalbuminuria (> $200 \mu \mathrm{g} / \mathrm{min}$ ).

degrades type IV collagen the accumulation of which is a central and precocious event in the development of diabetic glomerular extracellular matrix abnormalities. Thus, as type IV collagen metabolism is abnormal in diabetic nephropathy, the MMP2 loop should have a pivotal position in this phenomenon since mechanisms regulating the steady-state level of collagen IV have to be triggered. To investigate whether in human diabetes an abnormal matrix degradation occurs and plays a role in the pathogenesis of diabetic nephropathy, we studied the expression of MMP2 and its specific inhibitor TIMP2 in the two main kidney compartments, glomeruli and tubulointerstitium, of NIDDM patients and compared results with two control groups, one with inflammatory nephropathies and one with normal kidneys.

\section{Subjects and methods}

Patients. According to previously detailed criteria 16 NIDDM patients ( 4 females) from an ongoing multicentre study of renal structural-functional relationships were consecutively enrolled [5]; criteria for selection were: 1 ) age less than 70 years; 2) age at diagnosis of NIDDM more than 40 years; 3 ) known duration of NIDDM more than 2 years; 4) serum creatinine less than $180 \mu \mathrm{mol} / \mathrm{l}$; 5) absence of other obvious renal diseases; 6) no secondary forms of hypertension.

On admission to the study three 24-h urine collections were performed and albumin excretion rate (AER) was measured. In patients on antihypertensive treatment therapy was withdrawn 3 to 5 days before and during admission. Patients' clinical data are listed in Table 1. Light and immunofluorescence microscopy was performed on coded sections. Based on the resultant overview of the renal tissues, histological analysis was classified in the following categories [5]: C I, normal or near-normal
Renal histology, C I, normal or near-normal renal structure; C II, typical diabetic nephropathology; C III, atypical patterns of renal injury.

ACEI, angiotensin converting enzyme inhibitor; $\mathrm{CaB}$, calcium blocker

renal structure; C II, typical diabetic nephropathology, i.e. established diabetic lesions with balanced severity of glomerular, tubulo-interstitial and arteriolar changes; C III, atypical patterns of renal injury, i. e. severe tubulo-interstitial and/or arteriolar hyalinosis and/or global glomerular sclerosis in the presence of absent or mild glomerular changes. Electron microscopy was also performed. None of the patients had light, immunofluorescence, or electron microscopy findings suggesting specific and typical glomerulopathies other than diabetic nephropathy or the patterns described above.

Control subjects. Normal kidney biopsies were obtained from 5 patients ( 2 females) with kidney cancer (age range 45-62 years). Five patients ( 3 females) with "inflammatory" (i.e. non-metabolic) nephropathies were also considered (2 IgA glomerulonephritis and 3 chronic kidney transplant rejection) (age range 26-54 years).

After obtaining written informed consent for the study, "non-surgical" patients underwent renal biopsy under ultrasound guidance with a 14-gauge needle. In nephrectomies for renal cancer, biopsies were obtained immediately before renal artery clamp, with the same type of 14-gauge needle, from sites remote from the tumour-bearing tissue.

Microdissection and RNA extraction. Immediately after biopsy, approximately $1 / 10$ of the specimen was kept in a physiological solution containing $100 \mathrm{U}$ of RNAsin (Perkin Elmer, Branch Burg, TX, USA) in ice. Three to 12 glomeruli and the corresponding tubulointerstitium were isolated under a stereomicroscope (Zeiss, Jena, Germany), and immediately put into RNAzolB solution (BIOTEX, Houston, TX, USA). Total RNA was extracted using the RNAzolB method with some minor modifications: glomeruli and tubulointerstitium were homogenised in $200 \mu$ l of RNAzolB solution by pipetting; the homogenate was added with 0.1 volume of chloroform, then shaken vigorously, kept in ice for $5 \mathrm{~min}$ and centrifuged at $14,000 \mathrm{~g}\left(4^{\circ} \mathrm{C}\right)$ for $20 \mathrm{~min}$. The aqueous phase was transferred to a fresh "high yeld nucleic acid recovery" tube (Robbins Scientific, Sunnyvale, CA, USA), added to an equal volume of 
isopropanol, stored for $45 \mathrm{~min}$ in ice and centrifuged for $20 \mathrm{~min}$ at $14,000 \mathrm{~g}\left(4^{\circ} \mathrm{C}\right)$. The supernatant was removed and the RNA pellet was washed with $70 \%$ ethanol. To obtain a purer RNA preparation for the subsequent enzymatic assay, an additional overnight precipitation step with 2 volumes of $100 \%$ ethanol at $-20^{\circ} \mathrm{C}$ was performed. The RNA pellet was dissolved in $10 \mu \mathrm{l}$ of Diethyl Pyrocarbonate (Sigma, St. Louis, MI, USA (DEPC) water. Five $\mu$ l of RNA was used for the spectrophotometric quantitation at 260 and $280 \mathrm{~nm}$ using a $50 \mu \mathrm{l}$ microcell (Perkin Elmer).

Reverse transcription (RT). We reverse transcribed $30 \mathrm{ng}$ of total RNA in a final volume of $20 \mu \mathrm{l}$, in the presence of $5 \mathrm{mmol} / \mathrm{l}$ $\mathrm{MgCl}_{2}, 1 \mathrm{mmol} / 1 \mathrm{dNTP}, 1 \mathrm{U} / \mu \mathrm{l}$ RNAse inhibitor, $2.5 \mathrm{U} / \mu \mathrm{l}$ MuLV Reverse Transcriptase (Perkin Elmer), 2.5 mol/1 Random Examers in buffer: $50 \mathrm{mmol} / 1 \mathrm{KCl}, 10 \mathrm{mmol} / \mathrm{l}$ Tris $\mathrm{HCl}$, $\mathrm{pH}$ 8.3. This reaction was carried out for $30 \mathrm{~min}$ at $42^{\circ} \mathrm{C}$, and $5 \mathrm{~min}$ at $99^{\circ} \mathrm{C}$ in a thermalcycler (M. J. Research, Watertown, MA, USA).

Polymerase chain reaction. An aliquot of $2.5 \mu \mathrm{l}$ of $\mathrm{RT}$ reaction was used to amplify the following genes in different tubes: MMP2, TIMP2, and the housekeeping gene glyceraldehyde 3phosphate dehydrogenase (G3PDH) as internal standard. The specific cDNA sequences were amplified using the following primers: for MMP2 residues 1800-1816 (sense strand) and 2228-2247 (antisense strand), amplification product of $447 \mathrm{bp}$ [6]; for TIMP2, 349-369 and 813-933, 585 bp [6]; for G3PDH, 71-96 and 1053-1030, 983 bp (Clontech, Palo Alto, CA, USA). Although the primers have been designed to span one or more introns within the genes, control negative reactions, without reverse transcriptase, were performed during the cDNA synthesis step in order to exclude genomic contamination. The specificity of PCR products was verified by Southern blot analysis with specific probes on RNAs extracted from surgical biopsies of nephrectomies for renal cancer. Briefly, following amplification, $20 \mu \mathrm{l}$ aliquots were analysed by $1 \%$ agarose gel electrophoresis in Tris-Borate/EDTA Electrophoresis (TBE) buffer and PCR products highlighted by ethidium bromide staining. After gel separation, DNA was transferred onto a nylon membrane (Hybond $\mathrm{N}^{+}$; Amersham, Aylesbury, UK) by means of alkali blotting $(0.6 \mathrm{~mol} / \mathrm{l} \mathrm{NaCl}, 0.4 \mathrm{~mol} / \mathrm{l}$ $\mathrm{NaOH}$ ). After transfer, the nylon membrane was soaked for $5 \mathrm{~min}$ in neutralising solution $(1.5 \mathrm{~mol} / \mathrm{l} \mathrm{NaCl}, 0.5 \mathrm{~mol} / \mathrm{l}$ Tris$\mathrm{HCl}, \mathrm{pH} 7.2,1 \mathrm{mmol} / \mathrm{l}$ EDTA) and cross-linked by exposure to ultra violet light. Specific DNA probes for MMP2 and TIMP-2 were obtained by PCR-amplifying the cDNA of the HT-1080 human fibrosarcoma cell line, and the respective sequences were verified by the dideoxynucleotide-chain-termination method. Probe labelling, membrane hybridisation and signal detection were performed by the ECL Direct System (Amersham) following the manufacturer's instructions. Signals were detected by blue-light-sensitive autoradiography film (Hyperfilm-ECL; Amersham).

To increase the specificity and the efficiency of the PCR reaction the "hot start" procedure was applied by the use of a Taq specific antibody (Ab) (Clontech). The amplification was carried out in a final volume of $50 \mu \mathrm{l}$ containing: $1.5 \mathrm{mmol} / \mathrm{l}$ $\mathrm{MgCl}_{2}, 0.2 \mathrm{mmol} / \mathrm{l} \mathrm{dNTP}, 2 \mathrm{U}$ Taq DNA polymerase (Perkin Elmer), from a freshly prepared 28:1 molar ratio mixture of Taq antibody plus Taq polymerase, $0.4 \mu \mathrm{mol} / 1$ primers in $50 \mathrm{mmol} / \mathrm{l} \mathrm{KCl}, 10 \mathrm{mmol} / \mathrm{l}$ Tris $\mathrm{HCl}, \mathrm{pH}$ 8. cDNAs were amplified according to the following conditions: $94^{\circ} \mathrm{C}$ for $45 \mathrm{~s}, 60^{\circ} \mathrm{C}$ for $45 \mathrm{~s}, 72^{\circ} \mathrm{C}$ for $2 \mathrm{~min}$.

Comparative RT/PCR kinetic analysis. Kinetic analysis of amplified products was applied to all samples for each gene to ensure that signals were derived only from the exponential phase of the amplification. cDNAs were submitted to the first 30 cycles of amplification, and an aliquot of $5 \mu \mathrm{l}$ from each sample was drawn for electrophoretic analysis; then, the tubes were submitted to two more cycles of amplification and one more 5- $\mu$ l aliquot was drawn. This procedure was repeated six times until it reached a total of 40 cycles. The PCR products obtained after 30, 32, 34, 36, 38 and 40 cycles were analysed by Polyacrilamide Gel Electrophoresis (PAGE) in $7 \%$ polyacrilamide gel, $3 \% \mathrm{C}$ with $5 \%$ glycerol at 150 constant voltage in $1 \mathrm{X} \mathrm{TBE}$ for $1.30 \mathrm{~h}$, visualised by ethidium bromide staining and photographed. To enhance band signals, gels were silver stained according to the following protocol: $10 \%$ ethanol for $5 \mathrm{~min}, 1 \% \mathrm{HNO}_{3}$ for $3 \mathrm{~min}$, rinse in distilled water, $12 \mathrm{mmol} / \mathrm{l}$ $\mathrm{AgNO}_{3}$ for $20 \mathrm{~min}$, rinse in distilled water, developing in $280 \mathrm{mmol} / 1 \mathrm{NaCO}_{3}$ and $0.019 \%$ formaldehyde until the desired staining reached, stop the development with $10 \%$ $\mathrm{CH}_{3} \mathrm{COOH}$ for $2 \mathrm{~min}$.

Statistical analysis. Data, given as number of positive samples for total number of analysed tissues, were evaluated by the chi-squared test for the analysis of frequencies.

\section{Results}

Results of the study are reported in Table 2 . Of 16 NIDDM patients, 2 were not considered since the G3PDH housekeeping gene was not amplified due to RNA degradation or less efficient RT reaction. In all other samples, the internal standard was always amplified and a clear signal was visible between 30 and 34 cycles of amplification. The amplifiability of G3PDH ensured that the RNAs in the samples were not degraded and that the efficiency of the RT reaction was comparable between samples.

Examples of RT/PCR analysis are shown in Figure 1 , in which the amplification products relative to the mRNAs studied at glomerular and tubulointerstitial level are reported. MMP2 gene expression is differently regulated in the two compartments in the diabetic kidney; in fact, its mRNA was detectable in the tubulointerstitium in 13 diabetic patients, but not in the corresponding glomeruli $\left(\chi^{2}=20.6 ; p=0.000\right)$ even at 40 cycles of amplification; in contrast, TIMP2 was expressed both in glomeruli and tubulointerstitium in almost all diabetic patients (13 out of 14 subjects) $\left(\chi^{2}=1.37\right.$ for both; N. S. $)$. In glomeruli from control kidneys and from patients with inflammatory glomerulopathy the presence of both MMP2 and TIMP2 mRNAs was observed in almost all investigated biopsies (frequence differences were not significant), and band signals were clearly appreciable between 32 and 36 cycles for both.

\section{Discussion}

In diabetic nephropathy, mesangial expansion is tightly related to the prognosis of diabetic patients since increased mesangial area is predictive of kidney 
failure. It is generally assumed that mesangial expansion in diabetes is due to the extracellular matrix accumulation. Indeed, the increased synthesis of extracellular matrix proteins, type I, III and IV collagens, laminin and fibronectin has been demonstrated; in particular the enhanced production of collagen is a central event in diabetic glomerular matrix expansion, type IV collagen being one of the earliest matrix proteins involved [7]. Moreover, in diabetic nephropathy, both experimental and clinical investigations have shown activation of the transforming growth factor- $\beta$ (TGF- $\beta$ ) loop, of which the fibrogenetic consequences are well known [7-9]. However, the regulation of mesangial matrix deposition is dynamic and involves not only synthetic, but also degradative processes. The degradation of extracellular mesangial matrix occurs through the activity of glomerular MMPs, many of which have been described [10]. They are enzymes secreted in a latent form in the extracellular matrix where they work degrading at least one of the components. Control of the activity of these enzymes occurs at three regulatory levels: MMP gene transcription, MMP proenzyme activation, and MMP inhibition by specific natural inhibitors (TIMPs) [10]. The hypothesis of abnormal matrix degradation in diabetic nephropathy has recently been addressed by experimental studies which demonstrated the inhibition of the degradative arm of matrix turn-over (see below); this possibly leads to deranged extracellular matrix homeostasis. This conclusion was expected also in reference to the inhibiting activity of TGF- $\beta$ on collagenases. In fact, TGF- $\beta$ is known to decrease the MMP activities, while increasing the concentration of their specific inhibitors TIMPs [11].

Although messenger quantitation and enzymatic activity may not be necessarily coincident, there are a number of examples demonstrating that there exists a strict correlation between the level of the mRNA and of the corresponding protein. Therefore, the present study seems to confirm that also in human diabetic nephropathy, the collagenolytic arm (at least, the MMP2) is defective. In fact, under our experimental conditions (i.e. at the conditions of PCR amplification which were reached, and with the quantity of starting RNA which was available for retrotranscription) it was not possible to detect any PCR product for the MMP2 mRNA at the glomerular level; this does not mean that the MMP2 gene is not expressed at all, but certainly it is expressed at a very low level. Since, under the same experimental conditions, in almost all NIDDM tubulo-interstitial samples, control tissues, and all renal biopsies from patients with inflammatory glomerulopathy, the MMP2 gene PCR products were clearly seen even at 32 cycles of amplification, we are convinced that the lack of MMP2 signals in glomeruli of NIDDM patients was not due to technical problems. So, we are confident that the
Table 2. MMP2 and TIMP2 gene expression in microdissected kidney biopsies

\begin{tabular}{|c|c|c|c|c|}
\hline & \multicolumn{2}{|c|}{ Glomerulus } & \multicolumn{2}{|c|}{ Tubulo-interstitium } \\
\hline & MMP2 & TIMP2 & MMP2 & TIMP2 \\
\hline NIDDM & $0 / 14$ & $13 / 14$ & $13 / 14$ & $13 / 14$ \\
\hline Control kidneys & $4 / 5$ & $5 / 5$ & $5 / 5$ & $5 / 5$ \\
\hline $\begin{array}{l}\text { Inflammatory } \\
\text { glomerulopathies }\end{array}$ & $5 / 5$ & $4 / 5$ & $4 / 5$ & $4 / 5$ \\
\hline $\begin{array}{l}\text { Chi-square } \\
p \text { value }\end{array}$ & $\begin{array}{l}20.6 \\
0.000\end{array}$ & $\begin{array}{l}1.37 \\
\text { n.s. }\end{array}$ & $\begin{array}{l}1.37 \\
\text { n.s. }\end{array}$ & $\begin{array}{l}1.37 \\
\text { n.s. }\end{array}$ \\
\hline
\end{tabular}

Data are number of positive samples for total number of analysed tissues. In all analysed tissues, internal standard for G3PDH was amplifiable starting from 30-34 cycles of amplification

MMP2 gene is down-regulated in glomeruli from NIDDM patients. Concerning the TIMP2 gene, this was expressed either at the glomerular and tubulointerstitial level, irrespective of the pathological condition.

MMP2 and its inhibitor TIMP2 are constitutively expressed by renal cells in culture $[12,13]$, in mesangial cells in normal kidneys [14] and by macrophages and leukocytes that may infiltrate glomeruli during inflammatory diseases and allograft rejection. Thus, the observation that these genes were expressed in glomerular and tubular-interstitial tissues from control subjects and non-diabetic patients was expected. Due to ethical problems, we have no normal tissues from kidney transplants, probably the best samples to be used as controls. However, histological observation of the tissues on which RT/PCR analysis was performed and which were obtained from nephrectomies for kidney cancers showed normal aspects, with no inflammatory response. Furthermore, the very short time period between the general anaesthesia and the biopsy excludes the possibility of any interference on the MMP2 gene transcription by some drugs. Hence we believe these tissues may be considered as normal tissues, so that the MMP2 and TIMP2 gene expression in these control biopsies may be considered the "normal pattern".

The completely different pattern observed in NIDDM patients, i.e. the down-regulation in glomeruli of the MMP2 gene, is very interesting and seems to be distinctive of the diabetic milieu. Indeed, in vitro, in human mesangial cell cultures grown in high glucose concentrations, down-regulation of MMP2 has been demonstrated, while TIMP2 gene expression was modestly affected [13]. These results are congruous with those reported by Leehey et al. [1], i.e. the reduction of the gelatinolytic activity and the down-regulation of the MMP2 gene in rat mesangial cells in high glucose. In experimental in vivo diabetic models contradictory results have been reported concerning MMP2 gene expression at the glomerular level; in fact, while McLennan et al. [4] showed the early 


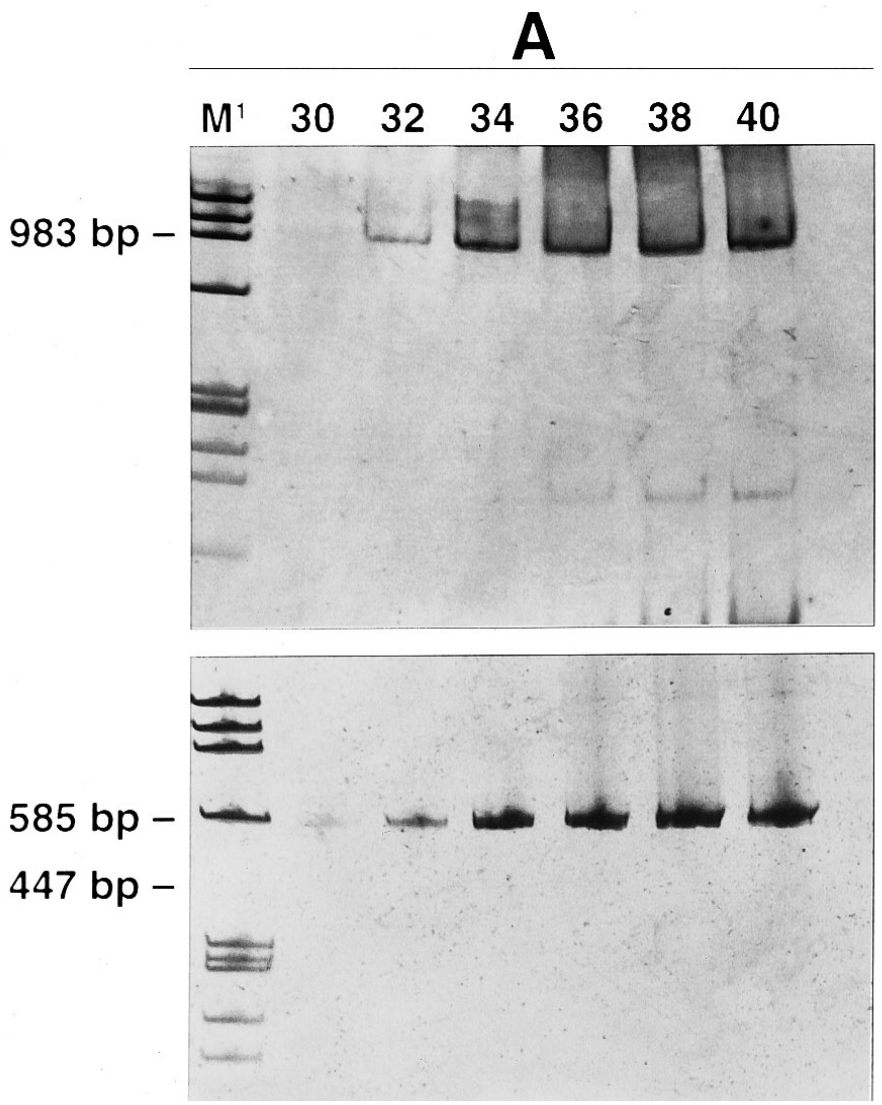

Fig. 1. PAGE analysis of the RT/PCR products generated by sequential cycles of amplification (30 to 40). The typical pattern of expression of MMP2 and TIMP2 genes plus G3PDH in microdissected glomeruli (A) and the corresponding tubulointerstitium (B) in an NIDDM patient is shown. TIMP2 and MMP2 PCT products were loaded in the same lane for comparison.

M: PBR 322/Hae III marker

$\mathrm{M}_{1}: \Phi X 174 /$ Hae III marker

disappearance of MMP2 gene expression, no alteration was shown by Nakamura et al. [15]. The latter have also demonstrated the reduction of glomerular MMP1 and MMP3 mRNA levels [15]; moreover, the decreased activity of cathepsin and MMPs in rats, namely a $117 \mathrm{kDa}$ MMP, has been observed by zymography to be most involved at the glomerular level [2]. Shankland and Scholey [16] in the streptozotocin rat model of diabetes have also demonstrated the up-regulation of TIMP (it is unclear which one) in the glomerulus, and, although no evaluation of MMP activities was carried out, the increase in TIMP was suggested to lead to reduced remotion of mesangial matrix. Finally, Reckelhoff et al. [3] have recently reported that, contrary to streptozotocin induced diabetes, a model in which mesangial expansion occurs, in the spontaneously diabetic BB/DP rat, in which there is no mesangial expansion, the glomerular activity of MMP (it was not specified which one) as

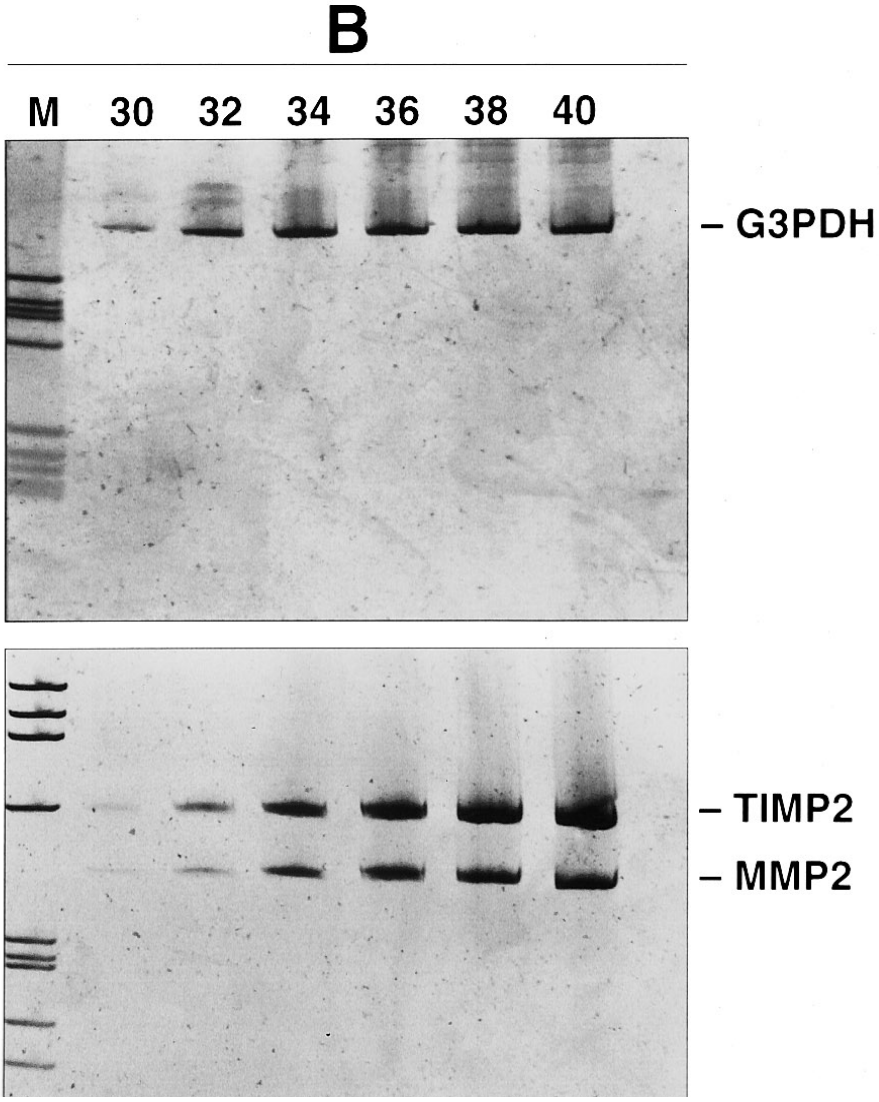

evaluated by zymography was normal. These results were interpreted as a demonstration that the different susceptibility to mesangial matrix enlargement in the two models is related to different modulation by the diabetic milieu of MMP activities.

Thus, from these experimental data the hypothesis was proposed that an important role in the pathogenesis of diabetic nephropathy is played by less efficient degradative matrix processes due to the diabetic milieu.

Our in vivo human data are very similar to the experimental data, and in particular to those presented by Leehey et al. [1], McLennan et al. [4], and Caenazzo et al. [13]. MMP2 in glomeruli, in human diabetes, is "universally" down-regulated, irrespective of histological classification, AER level, duration of diabetes, metabolic control of the disease, and blood pressure value. In other words, no relationship seems to exist between MMP2 gene expression and the gravity of the diabetic renal disease. In fact, MMP2 was down-regulated, while TIMP2 was still expressed in glomeruli in patients without any clinical or histological sign of diabetic nephropathy. The classification of AER in these patients may be queried because of the possible reduction of albuminuria by antihypertensive drugs; as a consequence of this a reclassification of patients on the basis of higher AERs would lead to a worse clinical picture of the patients enrolled in this study. However, the possibility that due to a patient selection bias, only individuals at 
high risk for diabetic nephropathy were recruited, thus explaining the "universal" down-regulation of MMP2 in glomeruli, seems to us unlikely because of the general criteria used for patient selection [5], and because of the heterogeneous clinical presentation, according to different clinical and morphological data of the enrolled patients. Thus, the present data sound a word of caution about the importance of this MMP in the pathogenesis of diabetic nephropathy.

From this study, however, it cannot be ruled out that different MMP systems play a more important role in the pathogenesis of human diabetic nephropathy as demonstrated in the diabetic rat by Nakamura et al. [15]; this aspect certainly deserves to be investigated. Although a certain overlap exists between different MMPs in regard to the extracellular matrix proteins degraded [14], so that one can also suspect that other MMP family members substitute functionally for each other, on the whole the experimental data $[2,3,15]$ seem to support the idea of a general failure at the glomerular level in diabetes of the degradative enzymes of the extracellular matrix.

In conclusion, this study demonstrates that: 1) in glomeruli of NIDDM patients the MMP2 gene is down-regulated; since TIMP2 is normally expressed in the glomerulus, a degradative imbalance of type IV collagen may be forwarded, confirming experimental findings.

2) In glomeruli of NIDDM patients the MMP2/ TIMP2 pattern is peculiar to NIDDM, since it is quite different from that observed in inflammatory glomerulopathies.

3) Since MMP2 gene down-regulation is observed in all NIDDM patients, irrespective of the level of the AER, and of renal histology classification, it seems a molecular epiphenomenon of diabetes, rather than a marker of diabetic nephropathy; furthermore, a peculiar susceptibility to the diabetic milieu of glomerular cell vs tubular cell MMP2 is obvious, the reason being unknown.

Thus, the MMP2/TIMP2 imbalance might be a necessary, but not sufficient condition for the pathogenesis of diabetic nephropathy in NIDDM.

Acknowledgements. This study was supported by Italian MURST $40 \%$ funds, and by the European Community through BIOMED-1 "Concerted action on alterations of extracellular matrix components in diabetic nephropathy and other glomerular diseases". We are grateful to Dr. Maurizio Onisto, Institute of Histology, University of Padova, for the kind gift of MMP2 and TIMP2 primers. The help of Dr. Signorelli, Division of Urology, Dolo-Venice Hospital, and Dr. Scutari, Division of Urology, Castelfranco-Treviso Hospital is also acknowledged.

\section{References}

1. Leehey DJ, Song RH, Alavi N, Singh AK (1995) Decreased degradative enzymes in mesangial cells cultured in high glucose media. Diabetes 44: 929-935

2. Reckelhoff JF, Tygart VL, Mitias M, Walcott JL (1993) STZ-induced diabetes results in decreased activity of glomerular cathepsin and metalloproteinase in rats. Diabetes 42: 1425-1532

3. Reckelhoff JF, Tygart VL, Racusen LC, Dzielak DJ (1994) Glomerular metalloproteinase activity in streptozotocintreated rats and in spontaneously diabetic rats $(\mathrm{BB} / \mathrm{DP})$. Life Sci 55: 941-950

4. McLennan SV, Fisher EJ, Yue DK, Turtle JR (1994) High glucose concentration causes a decrease in mesangium degradation. A factor in the pathogenesis of diabetic nephropathy. Diabetes 43: 1041-1045

5. Fioretto P, Maurer M, Brocco E, et al. (1996) Patterns of renal injury in NIDDM patients with microalbuminuria. Diabetologia 39: 1569-1576

6. Onisto M, Riccio MP, Scannapieco P, et al. (1995) Gelatinase A/TIMP2 imbalance in lymphonode-positive breast carcinomas, as measured by RT/PCR. Int J Cancer 63: 621-626

7. Sharma K, Guo I, Jin Y, Ericksen M, Ziyadeh FN (1994) Anti-TGF- $\beta$ antibody attenuates renal hypertrophy and matrix expansion in diabetic mice. J Am Soc Nephrol 5: 972-978

8. Yamamoto T, Nakamura T, Noble NA, Ruoslahti E, Border WA (1993) Expression of transforming growth factor $\beta$ is elevated in human and experimental diabetic nephropathy. Proc Natl Acad Sci USA 90: 1814-1818

9. Iwano M, Kubo A, Nishino T, et al. (1996) Quantification of glomerular TGF- $\beta 1$ mRNA in patients with diabetes mellitus. Kidney Int 49: 1120-1126

10. Matrisian LM (1990) Metalloproteinases and their inhibitors in matrix remodelling. Trends in Genetics 6: 121-125

11. Sharma K, Ziyadeh FN (1994) The emerging role of transforming growth factor- $\beta$ in kidney diseases. Am J Physiol 266: F829-F842

12. Martin J, Knowlden J, Davies M, Williams JD (1994) Identification and independent regulation of human mesangial cell metalloproteinases. Kidney Int 46: 877-885

13. Caenazzo C, Garbisa S, Onisto M, Zampieri M, Baggio B, Gambaro G (1997) Effect of glucose and heparin on mesangial $\alpha 1$ (IV)COLL and MMP-2/TIMP-2 mRNA expression. Nephrol Dial Transplant 12: 443-448

14. Davies M, Martin J, Thomas GJ, Lovett DH (1992) Proteinases and glomerular matrix turnover. Kidney Int 41: 671678

15. Nakamura T, Fukui M, Ebihara I, Osada S, Tomino Y, Koide H (1994) Abnormal gene expression of matrix metalloproteinases and their inhibitor in glomeruli from diabetic rats. Renal Physiol Biochem 17: 316-325

16. Shankland SJ, Scholey JW (1993) Expression of tissue inhibitor of metalloproteinase (TIMP) in glomeruli of normal and diabetic rats. J Am Soc Nephrol 4: 802 (Abstract) 